\title{
Dopaminergic Modulation of Human Intertemporal Choice: A Diffusion Model Analysis Using the D2-Receptor Antagonist Haloperidol
}

\author{
${ }^{\circledR}$ Ben Wagner, ${ }^{1}{ }^{\circledR}$ Mareike Clos, ${ }^{2}$ Tobias Sommer, ${ }^{2}$ and ${ }^{\circledR}$ Jan Peters ${ }^{1,2}$ \\ ${ }^{1}$ Department of Psychology, Biological Psychology, University of Cologne, Cologne 50969, Germany, and ${ }^{2}$ Department of Systems Neuroscience, \\ University-Medical-Center Hamburg-Eppendorf, Hamburg 20246, Germany
}

The neurotransmitter dopamine is implicated in diverse functions, including reward processing, reinforcement learning, and cognitive control. The tendency to discount future rewards over time has long been discussed in the context of potential dopaminergic modulation. Here we examined the effect of a single dose of the D2 receptor antagonist haloperidol ( $2 \mathrm{mg})$ on temporal discounting in healthy female and male human participants. Our approach extends previous pharmacological studies in two ways. First, we applied combined temporal discounting drift diffusion models to examine choice dynamics. Second, we examined dopaminergic modulation of reward magnitude effects on temporal discounting. Hierarchical Bayesian parameter estimation revealed that the data were best accounted for by a temporal discounting drift diffusion model with nonlinear trialwise drift rate scaling. This model showed good parameter recovery, and posterior predictive checks revealed that it accurately reproduced the relationship between decision conflict and response times in individual participants. We observed reduced temporal discounting and substantially faster nondecision times under haloperidol compared with placebo. Discounting was steeper for low versus high reward magnitudes, but this effect was largely unaffected by haloperidol. Results were corroborated by model-free analyses and modeling via more standard approaches. We previously reported elevated caudate activation under haloperidol in this sample of participants, supporting the idea that haloperidol elevated dopamine neurotransmission (e.g., by blocking inhibitory feedback via presynaptic D2 auto-receptors). The present results reveal that this is associated with an augmentation of both lower-level (nondecision time) and higher-level (temporal discounting) components of the decision process.

Key words: computational modeling; decision making; dopamine; haloperidol; intertemporal choice; pharmacology

Significance Statement

Dopamine is implicated in reward processing, reinforcement learning, and cognitive control. Here we examined the effects of a single dose of the $\mathrm{D} 2$ receptor antagonist haloperidol on temporal discounting and choice dynamics during the decision process. We extend previous studies by applying computational modeling using the drift diffusion model, which revealed that haloperidol reduced the nondecision time and reduced impulsive choice compared with placebo. These findings are compatible with a haloperidol-induced increase in striatal dopamine (e.g., because of a presynaptic mechanism). Our data provide novel insights into the contributions of dopamine to value-based decision-making and highlight how comprehensive modelbased analyses using sequential sampling models can inform the effects of pharmacological modulation on choice processes.

Received Mar. 11, 2020; revised Sep. 3, 2020; accepted Sep. 9, 2020.

Author contributions: B.W. and J.P. contributed unpublished reagents/analytic tools; B.W. and J.P. analyzed data; B.W. wrote the first draft of the paper; B.W. and J.P. wrote the paper; M.C., T.S., and J.P. designed research; M.C. performed research; M.C. and T.S. edited the paper.

This work was supported by Deutsche Forschungsgemeinschaft PE1627/5-1 to J.P. and S0952/3-1 to T.S. The authors declare no competing financial interests.

Correspondence should be addressed to Ben Wagner at ben.jonathan.wagner@uni-koeln.de or Jan Peters at jan.peters@uni-koeln.de.

https://doi.org/10.1523/JNEUROSCI.0592-20.2020

Copyright $\odot 2020$ the authors

\section{Introduction}

Future rewards are discounted in value (Peters and Büchel, 2011) such that humans and many animals prefer smaller-sooner (SS) rewards over larger-but-later (LL) rewards (temporal discounting). Steep discounting of reward value is associated with a range of maladaptive behaviors ranging from substance use disorders (Bickel et al., 2014), attention-deficit hyperactivity disorder (Jackson and MacKillop, 2016), and obesity (Amlung et al., 2016) to behavioral addictions, such as gambling disorder (Wiehler and Peters, 2015). Temporal discounting has thus been suggested to constitute a transdiagnostic process (Amlung et al., 
Table 1. Demographic and working memory data ${ }^{a}$

\begin{tabular}{|c|c|c|c|}
\hline & Placebo & Haloperidol & Group comparison \\
\hline Age (yr) & $24.4 \pm 3.4$ & $23.3 \pm 2.5$ & $t_{(45,614)}=1.40, p=0.17$ \\
\hline $\operatorname{Sex}(M / F)$ & $7 / 19$ & $6 / 17$ & $\chi_{(1)}^{2}=0.001, p=1$ \\
\hline WM baseline (z score) & $-0.0453 \pm 0.665$ & $0.0943 \pm 0.556$ & $t_{(46,826)}=-0.80, p=0.43$ \\
\hline Weight $(\mathrm{kg})(\mathrm{M} / \mathrm{F})$ & $70.7 \pm 3.39 / 63.5 \pm 3.19$ & $80.5 \pm 2.80 / 62.5 \pm 2.32$ & $t_{(36,702)}=-0.68, p=0.50$ \\
\hline
\end{tabular}

${ }^{a}$ Data are mean \pm SD.

2019; Lempert et al., 2019) with relevance for many psychiatric conditions.

Dopamine (DA) plays a central role in addiction (Robinson and Berridge, 1993). In rodents, reductions versus moderate increases in DA transmission led to increases and decreases in discounting, whereas the corresponding human literature is small and more heterogeneous (D'Amour-Horvat and Leyton, 2014). For example, de Wit et al. (2002) found that acute administration of D-amphetamine decreased impulsivity, such that temporal discounting was reduced under D-amphetamine. However, a later study did not replicate this effect (Acheson and de Wit, 2008). Administration of the D2/D3 receptor agonist pramipexole did not affect measures of impulsivity in another study $(n=10)$ from the same group (Hamidovic et al., 2008). In contrast, Pine et al. (2010) observed increased temporal discounting following administration of the catecholamine precursor L-DOPA compared with placebo in healthy control participants $(n=13)$, while the D2-receptor antagonist haloperidol did not modulate discounting. In a recent within-subjects study using L-DOPA in a substantially larger sample $(n=87)$, there was no overall effect on temporal discounting (Petzold et al., 2019). Rather, effects depended on baseline impulsivity, which the authors interpreted in the context of the inverted-U-model of DA effects on cognitive control functions (Cools and D'Esposito, 2011). Two recent studies have reported a reduction in discounting following administration of the selective D2/D3-receptor antagonist amisulpride (Weber et al., 2016) as well as the D2 receptor antagonist metoclopramide (Arrondo et al., 2015). Although the latter is primarily used clinically for its peripheral effects, it can pass the blood-brain barrier and act centrally (Shakhatreh et al., 2019).

A similar heterogeneity is evident when considering modelbased reinforcement learning (RL) (Doll et al., 2012), which in some studies (Shenhav et al., 2017), but not others (Solway et al., 2017), was associated with reduced temporal discounting. However, in contrast to temporal discounting (see above), LDOPA instead increased reliance on model-based RL in healthy controls (Wunderlich et al., 2012) and Parkinson's disease patients (Sharp et al., 2016). Notably, this overall effect was not observed in a recent study in a substantially larger sample ( $n=65)$ (Kroemer et al., 2019). Here, increased model-based RL under L-DOPA was restricted to participants with high working memory capacity.

One well-replicated behavioral effect in temporal discounting (magnitude effect) refers to the observation that the rate of temporal discounting decreases with increasing reward magnitude (Green et al., 1997). In humans, this effect depends on lateral PFC processing (Ballard et al., 2017); and in rodents, D-amphetamine effects on temporal discounting are more pronounced for large-magnitude conditions (Krebs et al., 2016). However, it is unclear whether DA impacts the magnitude effect in humans.

In the present study, we examined these processes using a between-subjects double-blind placebo-controlled pharmacological study with the D2-receptor antagonist haloperidol (2 mg). We previously reported increased dorsal striatal activation under haloperidol versus placebo in these participants (Clos et al., 2019a,b), compatible with a predominantly presynaptic effect of haloperidol that increases striatal dopaminergic signaling. Importantly, we extended previous pharmacological studies by applying a temporal discounting modeling framework based on a combination of discounting models with the drift diffusion model (DDM) (Pedersen et al., 2017; Fontanesi et al., 2019; Shahar et al., 2019; Peters and D'Esposito, 2020), allowing us to comprehensively examine drug effects on response time (RT) components related to both valuation and non-valuation-related processes.

\section{Materials and Methods \\ Participants}

Fifty-four healthy participants were initially enrolled in the study. Participants were screened by a physician for current diseases and current intake of prescription drugs or drugs of abuse. All participants were presently in good health and had no history of neurologic or psychiatric disorder with no current intake of prescription medication. Only healthy subjects were allowed to participate. Twenty-seven participants were randomly assigned to each group (placebo/haloperidol). Two participants from the haloperidol group did not complete the temporal discounting task. Technical problems led to working memory data loss from 4 participants ( 3 from the haloperidol, 1 from the placebo group), but these participants were still included in the temporal discounting data analysis.

Following filtering of RTs (see below; the fastest and slowest $2.5 \%$ of trials were excluded per participant), we examined the individual RT histograms for each subject (see Extended Data Fig. 1-1). This revealed that, even after filtering, the 3 participants with the fastest minimum RTs ( 2 from the haloperidol group and 1 from the placebo group) still showed implausibly fast responses on a number of trials (minimum RTs of 2, 2, and $234 \mathrm{~ms}$, in Subjects 24, 25, and 41, respectively) such that the minimum RTs were substantially faster than those in the remaining participants (all $\min (\mathrm{RT}) z$ scores of $-2.04,-2.04$, and -1.7 ; see Extended Data Fig. 1-2). These subjects were therefore excluded from further modeling.

We verified that there were no significant differences in demographic background in terms of age or baseline working memory capacity (Table 1). Potential side effects of the medication were monitored via multiple blood pressure and pulse measurements and evaluated via mood questionnaires. These analyses did not reveal significant group differences in terms of reported mood, side effects, or physiological parameters, as reported in our previous study (Clos et al., 2019b). Before enrollment, participants provided informed written consent, and all study procedures were approved by the local institutional review board (Hamburg Board of Physicians).

\section{Experimental design}

General procedure. The study consisted of two testing sessions performed on separate days. On the first day (T0), participants completed a background screening and a set of working memory tasks (see below). On the second day (T1), participants received either placebo or haloperidol $(2 \mathrm{mg})$. In line with the pharmacokinetics of haloperidol (Franken et al., 2017), testing on $\mathrm{T} 1$ was performed $5 \mathrm{~h}$ after drug administration to ensure appropriate plasma levels of haloperidol. During the first $2.5 \mathrm{~h}$, participants were under constant observation, and pulse as well as blood pressure levels were checked $30 \mathrm{~min}$ and $2 \mathrm{~h}$ after drug administration. 
During the waiting period, participants filled out questionnaires on current mood and medication effects. Participants then completed a number of unrelated tasks during an fMRI scanning session (total scan time 2.5 h.). Following scanning, they first completed the temporal discounting task outlined below, followed by a set of working memory tasks (digit span forward and backward, block span forward and backward, complex working memory span) (for detailed results, see Clos et al., 2019b).

Temporal discounting task. Participants performed 210 trials of a temporal discounting task where on each trial they made a choice between an SS reward available immediately and an LL reward. SS and LL rewards were randomly displayed on the left and right sides of the screen, and participants were free to make their choice at any time. For half the trials, the SS reward consisted of $20 €$; and for the remaining trials, the SS reward was fixed at $100 €$. These trials were presented randomly intermixed. LL options were computed via all combinations of a set of LL reward amounts (constructed by multiplying the SS reward with $[1.01,1.02,1.05,1.10,1.20,1.50,1.80,2.50,2,3,4,5$, $7,10,13])$ and LL delays $(1,2,3,5,8,30,60 \mathrm{~d})$, yielding 105 trials in total per magnitude condition. As is typically the case for temporal discounting tasks investigating magnitude effects (Green et al., 1997), all choices were hypothetical.

\section{Computational modeling}

Temporal discounting model. We applied a simple single-parameter hyperbolic discounting model to describe how value changes as a function of delay (Mazur, 1987; Green and Myerson, 2004) as follows:

$$
S V\left(L L_{t}\right)=\frac{A_{t}}{1+\exp \left(k+s_{k} * I_{t}\right) * D_{t}}
$$

Here, $A_{t}$ is the numerical reward amount of the LL option on trial $t$, $D_{t}$ is the LL delay in days on trial $t$, and $I_{t}$ is an indicator variable that takes on a value of 0 for trials from the large-magnitude condition (SS amount $=100 €$ ) data and 1 for trials from the small-magnitude condition (SS amount $=20 €$ ). The model has two free parameters: $k$ is the hyperbolic discounting rate from the large-magnitude condition (modeled in log-space) and $s_{k}$ is a weighting parameter that models the degree of change in discounting for small versus large SS rewards (i.e., higher values in $s_{k}$ reflect a greater magnitude effect) (Green et al., 1997).

\section{Softmax action selection}

Softmax action selection models choice probabilities as a sigmoid function of value differences (Sutton and Barto, 1998) as follows:

$$
P(L L)_{t}=\frac{\exp \left(\left(\beta+s_{\beta} * I_{t}\right) * S V\left(L L_{t}\right)\right)}{\exp \left(\left(\beta+s_{\beta} * I_{t}\right) * S V\left(S S_{t}\right)\right)+\exp \left(\left(\beta+s_{\beta} * I_{t}\right) * S V\left(L L_{t}\right)\right)}
$$

Here, $S V$ is the subjective value of the risky reward according to Equation 1 and $\beta$ is an inverse temperature parameter, modeling choice stochasticity (for $\beta=0$, choices are random and as $\beta$ increases, choices become more dependent on the option values). $S V\left(S S_{t}\right)$ was fixed at 100 for the large-magnitude condition and fixed at 20 for the small-magnitude condition. $I_{t}$ is again the dummy-coded condition regressor, and $s_{\beta}$ models the magnitude effect on $\beta$.

\section{Temporal discounting DDMs}

To more comprehensively examine dopaminergic effects on choice dynamics, we additionally replaced Softmax action selection with a series of DDM-based choice rules. In the DDM, choices arise from a noisy evidence accumulation process that terminates as soon as the accumulated evidence exceeds one of two response boundaries. In the present setting, the upper boundary was defined as selection of the LL option, whereas the lower boundary was defined as selection of the SS option.

RTs for choices of the SS option were multiplied by -1 before model fitting. We furthermore used a percentile-based cutoff, such that, for each participant, the fastest and slowest $2.5 \%$ of trials were excluded from the analysis. We then first examined a null model $\left(\mathrm{DDM}_{0}\right)$ without any value modulation. Here, the RT on each trial $t$ is distributed according to the Wiener First Passage Time (wfpt) as follows:

$$
R T_{t} \sim w f p t\left(\alpha+s_{\alpha} * I_{t}, \tau+s_{\tau} * I_{t}, z+s_{z} * I_{t}, v+s_{v} * I_{t}\right)
$$

The parameter $\alpha$ models the boundary separation (i.e., the amount of evidence required before committing to a decision), $\tau$ models the nondecision time (i.e., components of the RT related to motor preparation and stimulus processing), $z$ models the starting point of the evidence accumulation process (i.e., a bias toward one of the response boundaries, with $z>0.5$ reflecting a bias toward the LL boundary, and $z<0.5$ reflecting a bias toward the SS boundary), and $\nu$ models the rate of evidence accumulation. For each parameter $x$, we also include a parameter $s_{x}$ that models the change in that parameter from the high-magnitude $(S S=100 €)$ to the low-magnitude $(S S=20 €)$ condition (coded via the dummy-coded condition regressor $\left.I_{t}\right)$.

As in previous work (Pedersen et al., 2017; Fontanesi et al., 2019; Peters and D'Esposito, 2020), we then set up temporal discounting diffusion models by making trialwise drift rates proportional to the difference in subjective values between options. First, we set up a linear modeling scheme $\left(\mathrm{DDM}_{\text {lin }}\right)$ (Pedersen et al., 2017) as follows:

$$
v_{t}=\left(v_{\text {coeff }}+s_{v_{\text {coeff }}} * I_{t}\right) *\left(S V\left(L L_{t}\right)-S V\left(S S_{t}\right)\right)
$$

Here, the drift rate on trial $t$ is calculated as the scaled value difference between the LL and SS rewards. As noted above, RTs for SS options were multiplied by -1 before model estimation, such that this formulation predicts SS choices whenever SV(SS) $>$ SV(LL) (the trialwise drift rate is negative) and predicts longest RTs for trials with the highest decision conflict (i.e., in the case of SV(SS) $=S V(L L)$ the trialwise drift rate is zero). We next examined a DDM with nonlinear trialwise drift rate scaling $\left(D_{D} M_{S}\right)$ that has recently been reported to account for the value dependency of RTs better than the $\mathrm{DDM}_{\text {lin }}$ (Fontanesi et al., 2019; Peters and D'Esposito, 2020). In this model, the scaled value difference from Equation 4 is additionally passed through a sigmoid function with asymptote $v_{\text {max }}$ as follows:

$$
\begin{aligned}
& v_{t}=S\left[\left(v_{\text {coeff }}+s_{v_{\text {coeff }}} * I_{t}\right) *\left(S V\left(L L_{t}\right)-S V\left(S S_{t}\right)\right)\right] \\
& S(m)=\frac{2 *\left(v_{\max }+s_{v_{\max }} * I_{t}\right)}{1+\exp (-m)}-\left(v_{\max }+s_{v_{\max }} * I_{t}\right)
\end{aligned}
$$

All parameters, including $v_{\text {coeff }}$ and $v_{\max }$, were again allowed to vary according to the reward magnitude condition, such that we included $s_{x}$ parameters for each parameter $x$ that were multiplied with the dummycoded condition predictor $I_{t}$ (see above).

\section{Hierarchical linear regression}

Here we used the median posterior $\log (\mathrm{k})$ parameter of each participant from the $\mathrm{DDM}_{\mathrm{S}}$ model (see above) to compute the discounted values for all LL options. We then computed the trialwise decision conflict as the absolute difference between the subjective value of the LL reward and the corresponding smaller sooner reward. To ensure that the intercept in the regression model corresponds to the RT for the lowest decision conflict and to account for the strongly skewed distribution of value differences, we took the inverse of the absolute difference in SS and discounted LL values in each trial. To further avoid numerical instabilities when taking the inverse of absolute differences $<1$ (high conflict, e.g., $S V(L L)=20.10 €, S S=20 €$ ), these value differences were capped at 1 before computing the inverse. We then ran a hierarchical linear regression model in JAGS with 1/RT (to account for the skewed RT distribution) as dependent variable and decision conflict (inverse of the absolute value difference) as a predictor.

\section{Statistical analyses}

Hierarchical Bayesian models. Models were fit to all trials from all participants using a hierarchical Bayesian modeling approach with 

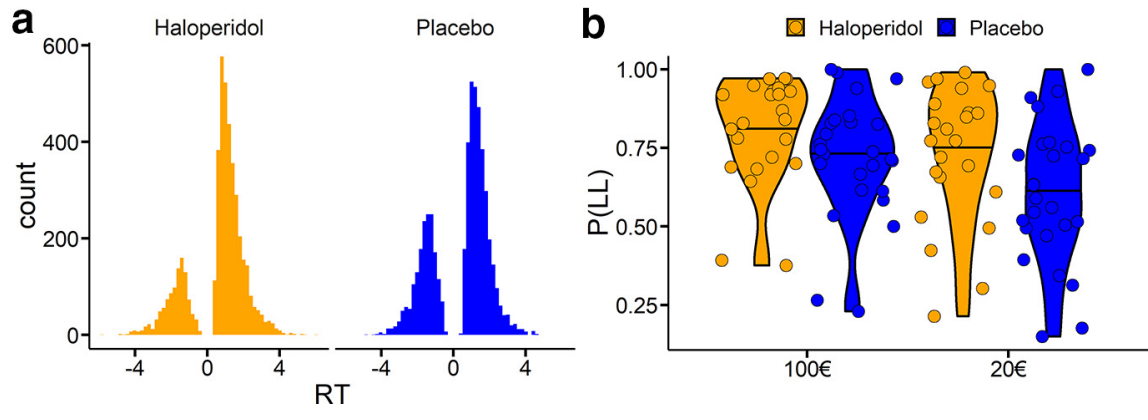

Figure 1. $\quad \boldsymbol{a}$, Overall RT distributions for the placebo group $(n=26)$ and the haloperidol group $(n=23)$. Negative RTs reflect choices of the SS option, whereas positive RTs reflect choices of the LL option. It can be seen that participants in the placebo group made numerically more SS selections than participants in the haloperidol group. For individual subject RT distributions, see Extended Data Figure 1-1. For minimum RTs following trial filtering, see Extended Data Figure 1-2. b, Proportion of LL choices per group and magnitude condition.

separate group-level distributions for all parameters for the placebo and haloperidol groups. Model fitting was performed using Markov Chain Monte Carlo as implemented in the JAGS software package (Plummer, 2003) (version 4.3) using the Wiener module for JAGS that implements the Wiener

First Passage Time (Wabersich and Vandekerckhove, 2014) (see Eq. 3) in combination with $\mathrm{R}$ (version 3.4) and the R2Jags package. For grouplevel means, we used uniform priors defined over numerically plausible parameter ranges (see Code and data availability). For all $s_{x}$ parameters modeling condition effects on model parameters, we used Gaussian priors with means of 0 and SDs of 2. For group-level precisions, we used $\gamma$ distributed priors $(0.001,0.001)$. We initially ran 2 chains with a burn-in period of 900,000 samples and thinning of two. Chain convergence was then assessed via the Gelman-Rubinstein convergence diagnostic $\hat{R}$ and sampling was continued until $1 \leq \hat{R} \leq 1.1$ for all group-level and individual-subject parameters. This occurred after a maximum of 1.3 million samples. For most parameters, $1 \leq \hat{R} \leq 1.01$ (Softmax: all parameters, $\mathrm{DDM}_{0}$ : all parameters, $\mathrm{DDM}_{\text {lin }}: 5$ parameters $1.01 \leq \hat{R} \leq 1.1, \mathrm{DDM}_{\mathrm{S}}: 9$ parameters $1.01 \leq \hat{R} \leq 1.1$ ). Relative model comparison was performed via the deviance information criterion (DIC), where lower values reflect a superior fit of the model (Spiegelhalter et al., 2002). A total of 10,000 additional samples were then retained for further analysis. We then show posterior group distributions for all parameters of interest as well as their $85 \%$ and $95 \%$ highest density intervals (HDIs). For group comparisons, we report Bayes factors (BFs) for directional effects Kass and Raftery, 1995 for the hyperparameter difference distributions of placebohaloperidol, estimated via kernel density estimation using $\mathrm{R}$ (version 4.01) via RStudio (version 1.3) interface. These are computed as the ratio of the integral of the posterior difference distribution from 0 to $\infty$ versus the integral from 0 to $-\infty$. Using common criteria (Beard et al., 2016), we considered BFs between 1 and 3 as anecdotal evidence, BFs $>3$ as moderate evidence, and $\mathrm{BFs}>10$ as strong evidence. $\mathrm{BFs}>30$ and $>100$ were considered as very strong and extreme evidence, respectively, whereas the inverse of these reflect evidence in favor of the opposite hypothesis.

Parameter recovery analyses. To ensure that the parameters underlying the data-generating process could be recovered using our modeling procedures, we performed posterior predictive checks for the best-fitting model $\left(\mathrm{DDM}_{\mathrm{S}}\right)$. During model estimation, we generated 10,000 datasets simulated from the posterior distribution of the $\mathrm{DDM}_{\mathrm{S}}$. Ten of these simulated datasets were randomly selected and refit with the $\mathrm{DDM}_{\mathrm{S}}$ (see previous section) (Fontanesi et al., 2019; Peters and D'Esposito, 2020). Parameter recovery was then assessed in two ways. For group-level parameters, we examined whether the estimated 95\% highest posterior density intervals contained the true generating parameters. For subjectlevel parameters, we examined scatter plots of generating versus estimated single-subject parameters, pooled across all 10 simulations.

Posterior predictive checks. To check whether the best-fitting model indeed captured key aspects of the data, in particular the value dependency for RTs, we performed posterior predictive checks (Peters and
D’Esposito, 2020) as follows. For each individual participant, we binned trials into five bins, according to the absolute difference in LL versus SS value ("decision conflict," computed according to each participant's median posterior $\log (\mathrm{k})$ parameter from the $\mathrm{DDM}_{\mathrm{S}}$, and separately for the high- and low-magnitude conditions). For each participant and condition, we then plotted the mean observed RTs as a function of decision conflict, as well as the mean RTs across 10,000 datasets simulated from the posterior distributions of the $\mathrm{DDM}_{0}, \mathrm{DDM}_{\text {lin }}$ and $\mathrm{DDM}_{\mathrm{S}}$.

\section{Code and data availability}

Model code is available on the Open Science Framework (https://osf.io/wm7ud/). Raw choice data are available from Zenodo.org (https://doi.org/10.5281/zenodo.4006531) for researchers meeting the criteria for access to confidential data.

\section{Results}

Subjective and physiological drug effects

As reported in detail in our previous papers (Clos et al., 2019a,b), there were no significant group differences with respect to reported side effects, subjective mood, heart rate, or blood pressure relative to baseline. Likewise, groups did not differ with respect to the actual and guessed drug condition (haloperidol vs placebo) (Clos et al., 2019b).

\section{Model free analysis of temporal discounting}

Figure $1 a$ shows the overall RT distributions per group with choices of the LL option coded as positive RTs and choices of the SS option coded as negative RTs. As a model-free measure of temporal discounting, we examined proportions of LL choices as a function of group (placebo vs haloperidol) and condition (100€ vs $20 €$ reference reward). Raw proportions of LL choices are plotted in Figure $1 b$. ANOVA on arcsine-square-root transformed proportion values with the within-subject factor magnitude (high [100€] vs low [20€] SS reward) and the betweensubject factor drug (placebo vs haloperidol) confirmed a significant magnitude effect $\left(F_{(1,47)}=96.86, p<0.001\right)$ such that participants overall made more LL selections in the high-magnitude condition. Furthermore, effects of drug $\left(F_{(1,47)}=3.47, p=0.068\right)$ and drug $\times$ magnitude $\left(F_{(1,47)}=3.31, p=0.075\right)$ showed trendlevel significance.

\section{Softmax choice rule}

First, we analyzed our data using a standard Softmax choice rule (Fig. 2). This analysis revealed an overall drug effect on $\log (\mathrm{k})$, such that discounting was substantially lower in the haloperidol group compared with the placebo group (Fig. 1a). Examination of BFs indicated that a decrease in $\log (\mathrm{k})$ in haloperidol versus placebo was $\sim 116$ times more likely than an increase (Table 2 ).

\section{Model comparison}

We next compared three versions of the DDM that varied in the way that they accounted for the influence of value differences on trialwise drift rates, based on the DIC (Spiegelhalter et al., 2002). In each model, we included separate group-level distributions for the two drug conditions (haloperidol vs placebo). Furthermore, for each parameter $x$, we included a shift parameter $s_{x}$ modeling the change in parameter $x$ from the high-magnitude condition (SS reward =100€) to the low-magnitude condition (SS 

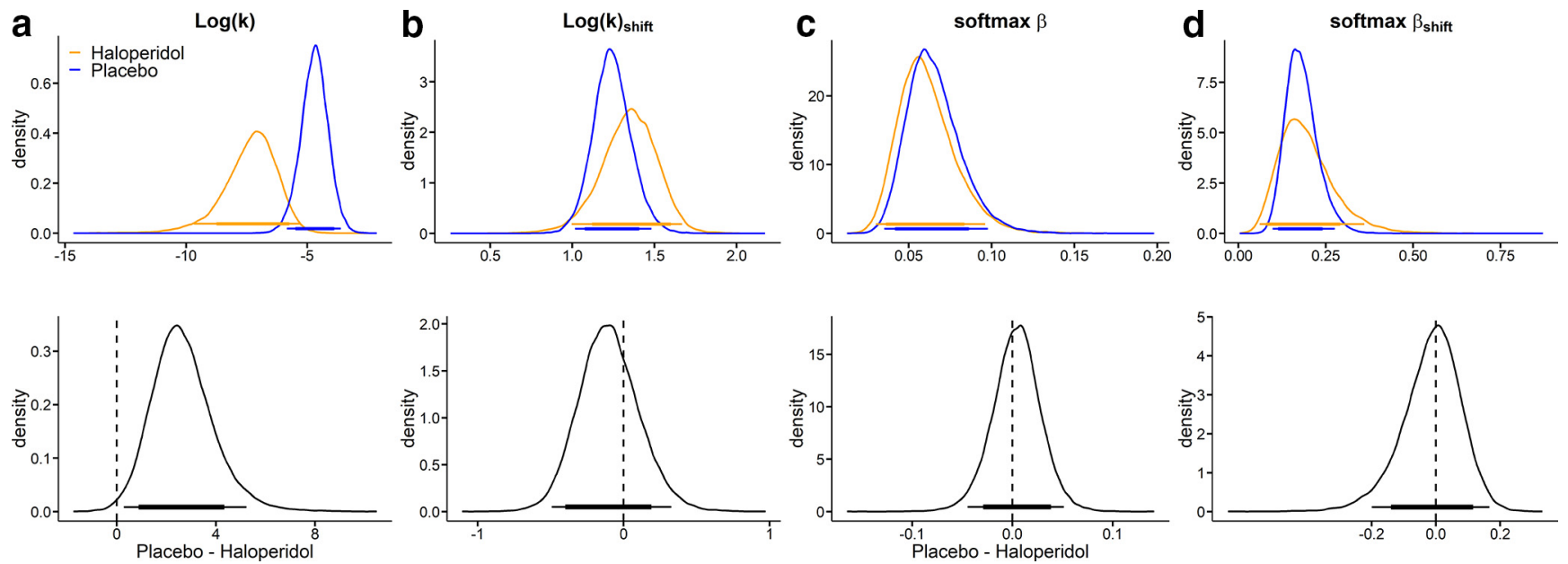

Figure 2. Modeling results (blue: placebo, orange: haloperidol) from a hierarchical Bayesian Model with softmax choice rule. $\boldsymbol{a}, \log (\mathrm{k})$ is the $\log ($ discount rate) from the high magnitude condition (smaller-sooner reward $=100 €) . \boldsymbol{b}, \log (\mathrm{k})_{\text {shift }}$ is the change in $\log (\mathrm{k})$ from the high magnitude condition to the low magnitude condition (smaller-sooner reward $\left.=20 €\right)$. $\boldsymbol{c}$, is the inverse temperature parameter. $\boldsymbol{d}_{\text {,shift }}$ the corresponding shift in inverse temperature from the high to low magnitude condition. The thin (thick) horizontal lines denote $95 \%$ (85\%) highest posterior density intervals.

Table 2. Summary of group differences in model parameters for the temporal discounting Softmax model ${ }^{a}$

\begin{tabular}{llllll}
\hline & \multicolumn{2}{l}{ Baseline } & & \multicolumn{2}{l}{ Magnitude effect } \\
\cline { 2 - 3 } \cline { 6 - 6 } Parameter & $\mathrm{M}_{\text {diff }}$ & $\mathrm{dBF}$ & & $\mathrm{M}_{\text {diff }}$ & $\mathrm{dBF}$ \\
\hline $\log (\mathrm{k})$ & 2.66 & 116.34 & & -0.10 & 0.42 \\
Temp & 0.03 & 1.36 & & -0.01 & 0.89 \\
\hline
\end{tabular}

${ }^{a}$ For each parameter, we report mean posterior group differences $\left(\mathrm{M}_{\text {diff }}\right)$ and $\mathrm{BFs}(\mathrm{dBF})$ testing for directional effects on both the baseline parameter in the $100 €$ condition (left columns) and on the magnitude effect on each parameter (right columns). BFs $<0.33$ reflect evidence for placebo $<$ haloperidol, whereas $\mathrm{BFs}>3$ reflect evidence for placebo $>$ haloperidol. For details, see Statistical analyses.

Table 3. Model comparison of three variants of the DDM based on the DIC (Spiegelhalter et al., 2002) where lower values indicate a better model fit ${ }^{a}$

\begin{tabular}{llll}
\hline & DIC & & \\
\cline { 2 - 4 } Model & Placebo & Haloperidol & Full model \\
\hline DDM $_{0}$ & 11792.1 & 10034.5 & 21833.8 \\
DDM $_{\text {lin }}$ & 10835.0 & 10092.1 & 20923.9 \\
DDM $_{\text {s }}$ & 8586.5 & 8161.7 & 16771.8 \\
\hline
\end{tabular}

${ }^{a}$ The data were generally better accounted for by a temporal discounting DDM with DDMs compared with $\mathrm{DDM}_{\text {lin }}$ and $\mathrm{DDM}_{0}$.

Table 4. Proportion of correctly predicted binary choices for each group and model $^{a}$

\begin{tabular}{lll}
\hline & Placebo & Haloperidol \\
\hline Softmax & $0.89(0.77-1.00)$ & $0.90(0.78-0.98)$ \\
DDM $_{0}$ & $0.73(0.57-1.00)$ & $0.80(0.60-0.98)$ \\
DDM $_{\text {lin }}$ & $0.88(0.71-0.97)$ & $0.85(0.62-0.98)$ \\
DDM $_{s}$ & $0.89(0.81-1.00)$ & $0.90(0.82-0.98)$ \\
\hline
\end{tabular}

${ }^{a}$ Data are mean (range).

reward $=20 €)$ (see Materials and Methods). These $s_{x}$ parameters were modeled with Gaussian priors with means of zero (see Materials and Methods). $\mathrm{DDM}_{0}$ assuming constant drift rates independent of value was also included and compared with two variants of the DDM using either linear $\left(\mathrm{DDM}_{\mathrm{lin}}\right)$ (Pedersen et al., 2017) or in a nonlinear (sigmoid) drift rate scaling (Fontanesi et al., 2019; Peters and D'Esposito, 2020). In both drug conditions as well as overall (Table 3 ), the data were best accounted for by a DDM with nonlinear drift rate scaling $\left(\mathrm{DDM}_{\mathrm{S}}\right)$.
We also compared the three diffusion models and the Softmax model with respect to the proportion of binary choices (LL vs SS selections) that they correctly accounted for. As can be seen from Table 4, the $\mathrm{DDM}_{\mathrm{s}}$ performed numerically on par with the Softmax model, whereas the $\mathrm{DDM}_{\text {lin }}$ performed slightly worse.

\section{Overall group differences}

We next examined overall group differences in model parameters for the baseline (SS reward $=100 €$ ) condition. Results are plotted in Figure 3, and BFs for all group comparisons are listed in Table 5. In both groups, there was a positive association between trialwise drift rates and value differences, as the $95 \%$ HDI for the drift rate coefficient parameter did not include 0 in either group (Fig. 3b). Likewise, there was a slight bias toward the SS option in both groups, as the 95\% HDI for bias was $<0.5$ in both cases (Fig. 3e).

We furthermore observed substantially lower group-level discount rates $\log (\mathrm{k})$ in the haloperidol group compared with placebo, such that the 95\% HDI of the posterior group difference in $\log (\mathrm{k})$ was $>0$ (Fig. $3 a$; Table 5). Interestingly, the nondecision time was likewise substantially lower in the haloperidol group (Fig. 3c; Table 5), amounting, on average, to $180 \mathrm{~ms}$ faster nondecision times.

\section{Magnitude effects on model parameters}

We next turned to the effects of the magnitude manipulation on diffusion model parameters, that is, the change in each parameter in the low-magnitude condition compared with the highmagnitude baseline condition. Results are plotted in Figure 4, and BFs for all directional group comparisons are listed in Table 5. There was a substantial magnitude effect on $\log (\mathrm{k})$, such that discounting was steeper in the low-magnitude condition (Fig. $4 a$ ). Interestingly, this pattern of results was not mirrored by in the magnitude effect on the starting point/bias parameter. Instead, the bias was shifted in the direction of a neutral bias (0.5) in the low-magnitude condition (Fig. 4e) in both groups. An additional interesting observation is that the nondecision time was increased in the low-magnitude condition by on average $\sim 30 \mathrm{~ms}$ (Fig. $4 c$ ). 

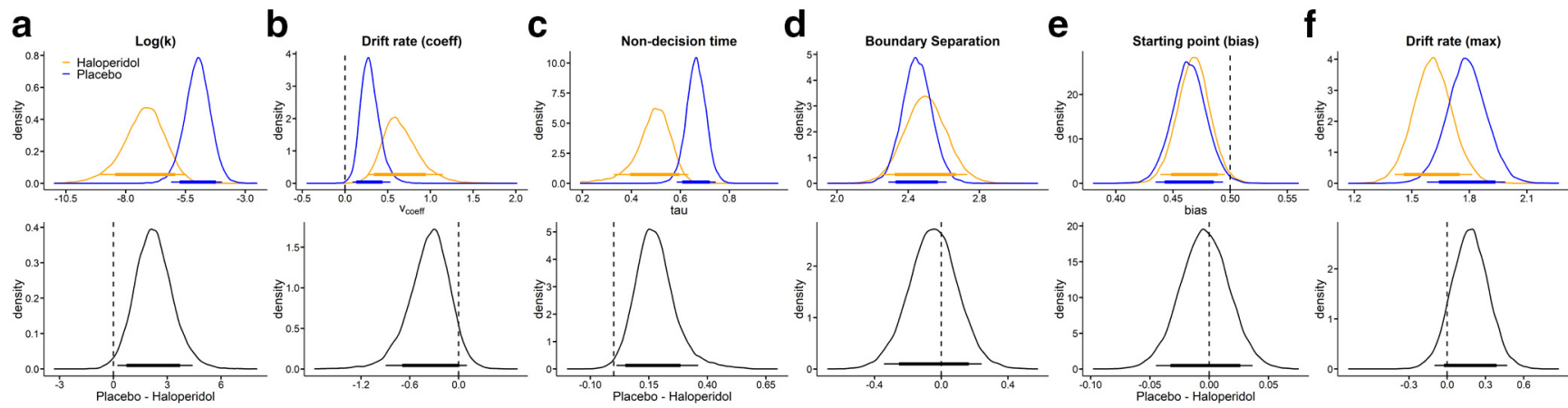

Figure 3. Posterior distributions (blue: placebo, orange: haloperidol) per parameter (top row: $\boldsymbol{a}, \log (\mathrm{k}) ; \boldsymbol{b}$, Drift rate coefficient; $\boldsymbol{c}$, Nondecision time; $\boldsymbol{d}$, Boundary separation; $\boldsymbol{e}$, Starting point bias; $\boldsymbol{f}$, Drift rate maximum) and group differences (bottom row, placebo-haloperidol) for the baseline condition (smaller-sooner reward = 100€). Thin (thick) horizontal lines denote $95 \%$ (85\%) highest posterior density intervals.

Table 5. Summary of group differences in model parameters for the temporal discounting $\mathrm{DDM}^{a}$

\begin{tabular}{lccccc}
\hline & \multicolumn{2}{l}{ Baseline } & & \multicolumn{2}{c}{ Magnitude effect } \\
\cline { 2 - 3 } \cline { 6 - 6 } Model parameter & $M_{\text {diff }}$ & $d B F$ & & $M_{\text {diff }}$ & $d B F$ \\
\hline Log $(k)$ & 2.26 & 77.9 & & -0.093 & 0.47 \\
Drift rate coefficient & -0.365 & 0.061 & & 0.020 & 2.73 \\
Nondecision time & 0.180 & 98.4 & & -0.0001 & 0.95 \\
Boundary separation & -0.047 & 0.60 & & 0.017 & 1.47 \\
Starting point bias & -0.004 & 0.74 & & -0.017 & 0.26 \\
Drift rate maximum & 0.18 & 8.27 & & 0.16 & 16.88 \\
\hline
\end{tabular}

${ }^{a}$ For each parameter, we report mean posterior group differences $\left(M_{\text {diff }}\right)$ and $B F s(d B F)$ testing for directional effects on both the baseline parameter in the $100 €$ condition (left columns) and on the magnitude effect on each parameter (right columns). BFs $<0.33$ reflect evidence for placebo $<$ haloperidol, whereas BFs $>3$ reflect evidence for placebo $>$ haloperidol. For details, see Statistical analyses.

Both drift rate components $\left(v_{\text {coeff }}\right.$ and $\left.v_{\max }\right)$ were increased in the $20 €$ condition (Fig. $4 b, f$ ). This overall effect might in part be attributable to the fact that, in the model, these two parameters effectively scale the trialwise value differences to the appropriate scale of the DDM (Pedersen et al., 2017). Because average value differences spanned a smaller absolute range in the $20 €$ condition, this is compensated in the model by increasing both $v_{\text {coeff }}$ (Fig. $4 b$ ) and $v_{\max }$ (Fig. 4f). Notably, under haloperidol, the drift rate coefficient was somewhat increased, whereas the maximum drift rate was attenuated. There might be some trade-off between the drift rate components, which could contribute to such contrasting effects, such that increases in one component can be compensated by decreases in the other. There was also some evidence for a reduced magnitude effect on the maximum drift rate (Fig. $4 f$ ) in the haloperidol group. This could be a reflection of the fact that the magnitude effect on LL choice proportions was numerically attenuated under haloperidol (Fig. 1a), leading to overall more homogeneous values in the two conditions. Difference distributions in the remaining model parameters were centered at zero, indicating no systematic group differences.

\section{Correlation of model parameters}

For descriptive purposes, we show the full correlation matrices for all single-subject median posterior parameters in Figure $5 a$ for haloperidol and Figure $5 b$ for placebo.

\section{Hierarchical linear regression}

We also explored whether the qualitative pattern of results could be reproduced using a hierarchical linear regression, modeling trialwise inverse RTs as a function of value differences (see Materials and Methods). Full posterior distributions of all parameters are shown in Figure 6. This analysis reproduced effects observed for the full DDM. For example, the slope was overall negative, reflecting the decrease in 1/RT for increasing conflict (Fig. 6a). The intercept was numerically smaller under haloperidol $(\mathrm{dBF}=0.11$; see Table 6$)$, mirroring the drug effect on the nondecision time in the $\mathrm{DDM}_{\mathrm{S}}$. However, a direct comparison with DDM parameters is complicated by the fact the intercept in the regression model also captures RT components that in the DDM are reflected in the boundary separation, as well as potentially additional nonlinear aspects of the evidence accumulation process that cannot be accounted for by the slope. These effects are visualized in Figure $6 e$ where we plot the 1/RT predicted by this regression model as a function of group, condition, and decision conflict. This illustrates again the slope effect in the baseline condition and the attenuated intercept under haloperidol.

\section{Associations with working memory span}

Exploratory analyses did not reveal associations between model parameters of interest $(\log (\mathrm{k})$, nondecision time, drift rate scaling) and working memory score (all $|r|<0.38$ ).

\section{Posterior predictive checks}

We next performed extensive posterior predictive checks to ensure that the best-fitting model $\left(D_{D} M_{S}\right)$ could account for RTs of individual participants in both groups. To this end, we binned the trials of each individual participant into five bins, according to the absolute difference in LL versus SS value (computed according to each participant's median posterior $\log (\mathrm{k}) \mathrm{pa}-$ rameter from the $\mathrm{DDM}_{\mathrm{S}}$ ). For each bin, participant, and condition, we then plot the mean observed RT, as well as the mean simulated RT across 10,000 datasets simulated from the posterior distributions of the $\mathrm{DDM}_{0}, \mathrm{DDM}_{\text {lin }}$, and $\mathrm{DDM}_{\mathrm{S}}$. These results are shown in Figure 7 for the placebo group and Figure 8 for the haloperidol group. As can be seen, the $\mathrm{DDM}_{\mathrm{S}}$ provided a much better account of how RTs vary as a function of decision conflict than the $\mathrm{DDM}_{\text {lin }}$ in the vast majority of participants in both groups. This was mainly because the $\mathrm{DDM}_{\text {lin }}$ overestimated RTs with medium decision conflict and underestimated RTs in cases of very low decision conflict (Peters and D'Esposito, 2020).

Some additional nontrivial patterns in the data deserve mention. For example, while the $\mathrm{DDM}_{\mathrm{S}}$ in most cases predicted longest RTs for choices with the highest decision conflict, this was not always the case (see, e.g., the low-magnitude condition of Participant 34 from the placebo group in Fig. 7). In this case, in the low-magnitude condition, the participant exhibited a 

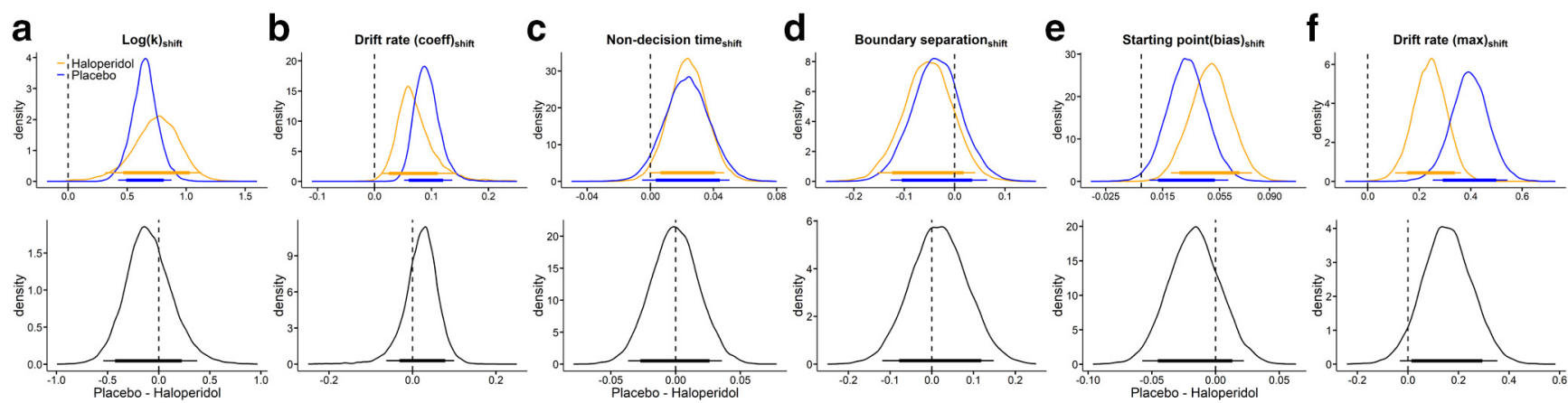

Figure 4. Posterior distributions (blue: placebo, orange: haloperidol) of the change in each parameter from the high magnitude (baseline) to the low magnitude condition (top row: $\boldsymbol{a}$, Log

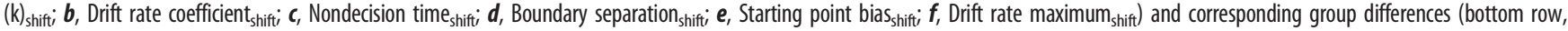
placebo-haloperidol). Thin (thick) horizontal line denote $95 \%$ (85\%) highest posterior density intervals.

a

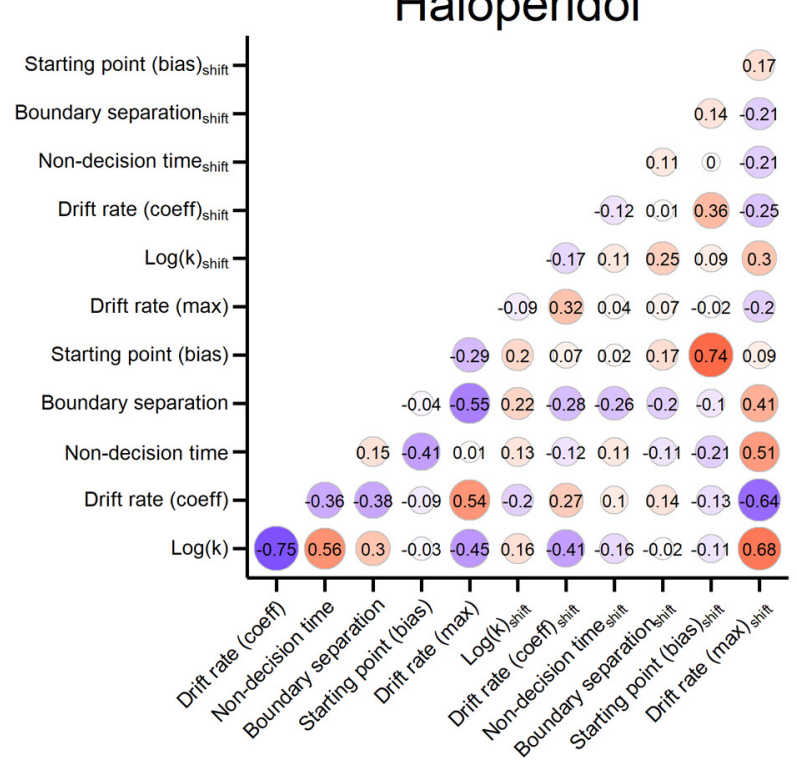

b

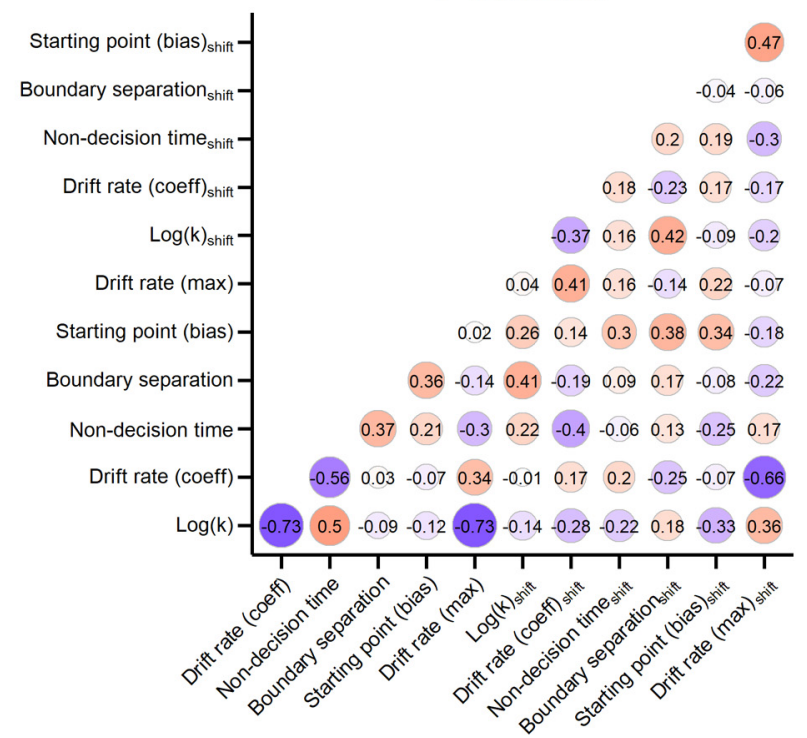

Figure 5. Correlations between all single-subject median posterior parameter estimates across participants from the haloperidol (a) and placebo group (b).

$\mathbf{a}$
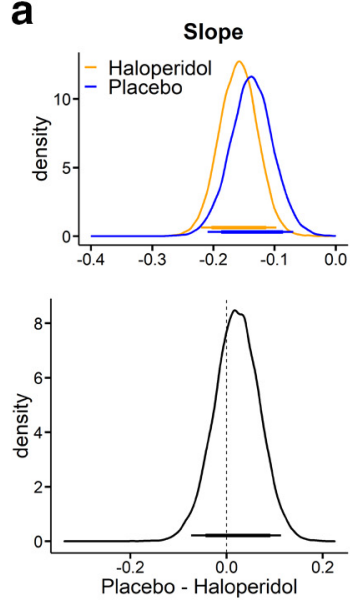

b
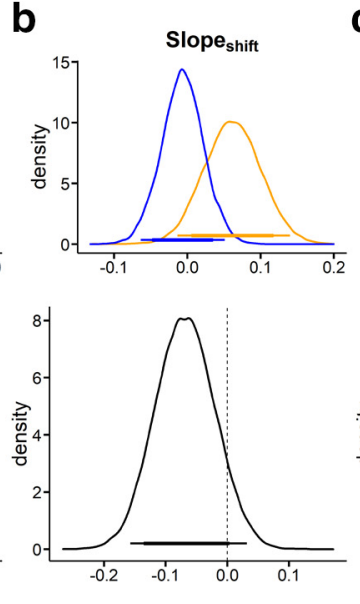

C
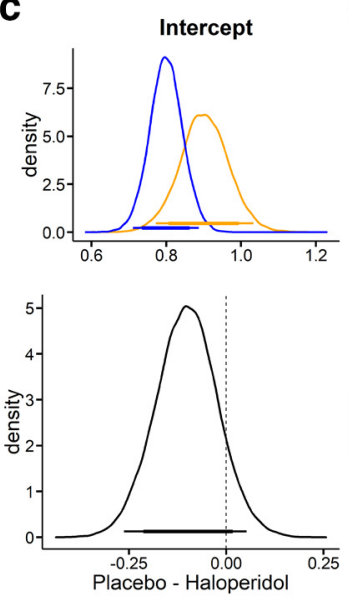

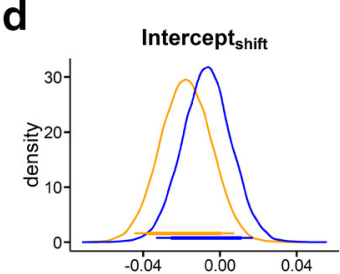

e
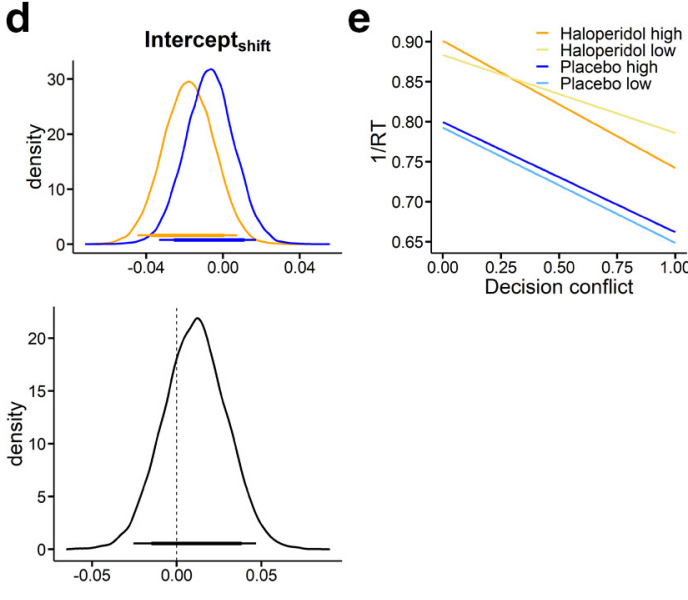

Figure 6. Modeling results (blue: placebo, orange: haloperidol) from a hierarchical linear regression with decision conflict as a predictor and 1/RT as dependent variable. Top row: The slope in $\boldsymbol{a}$, represents the influence of increasing decision conflict (decreasing value differences) on 1/RT. The intercept in c, here corresponds to 1/RT for the lowest decision conflict (highest subjective value difference) from the high magnitude condition (smaller-sooner reward $=100 €)$. Shift-parameters again reflect the change in slope and intercept $(\boldsymbol{b}, \boldsymbol{d})$ from the high to the low magnitude condition. $\boldsymbol{e}$, Illustrates 1/RT predicted by this regression model as a function of group, condition and decision conflict. Bottom row: Corresponding group differences (placebo-haloperidol). The thin (thick) horizontal lines denote $95 \%$ (85\%) highest posterior density intervals. 
Table 6. Summary of group differences in model parameters for the hierarchical linear regression model ${ }^{a}$

\begin{tabular}{|c|c|c|c|c|}
\hline \multirow[b]{2}{*}{ Model parameter } & \multicolumn{2}{|l|}{ Baseline } & \multicolumn{2}{|c|}{ Magnitude effect } \\
\hline & $M_{\text {diff }}$ & $d B F$ & $M_{\text {diff }}$ & $d B F$ \\
\hline Slope & 0.02 & 2.09 & -0.07 & 0.09 \\
\hline Intercept & -0.10 & 0.11 & 0.01 & 2.59 \\
\hline
\end{tabular}

${ }^{a}$ For each parameter, we report mean posterior group differences $\left(M_{\text {diff }}\right)$ and $B F s(d B F)$ testing for directional effects on both the baseline parameter in the $100 €$ condition (left columns) and on the magnitude effect on each parameter (right columns). BFs $<0.33$ reflect evidence for placebo $<$ haloperidol, whereas $\mathrm{BFs}>3$ reflect evidence for placebo $>$ haloperidol. For details, see Statistical analyses.

relatively small boundary separation (1.84) and drift rate coefficient (0.24), in combination with a bias toward the SS boundary $(0.43)$ and a high discount rate $\log (\mathrm{k})(-0.7)$. In such a constellation, the bias toward the SS boundary can only be overcome when value evidence is accumulated for a relatively long time (because $v_{\text {coeff }}$ is relatively small), giving rise to long RTs for LL choices (which in this case only occurred in the case of low decision conflict).

\section{Parameter recovery}

As a final model check, we ran a series of parameter recovery simulations. Here, we randomly selected 10 datasets simulated from the posterior distribution of the $\mathrm{DDM}_{\mathrm{S}}$ (see Materials and Methods), and refit these synthetic data with the $\mathrm{DDM}_{\mathrm{S}}$. Results are shown in Figure 9 for the baseline (high magnitude 100€) parameters, and Figure 10 for the parameters modeling condition effects. As can be seen from these plots, for both baseline and condition effects, this revealed that group-level parameters (Figs. 9,10 , bottom rows) recovered well, such that the true generating parameters were generally contained in the estimated 95\% HDIs.

Parameter recovery for individual-subject parameters was excellent for all baseline (100€ magnitude) parameters (Fig. 9, top row) such that the correlation between generating and estimated individual-subject parameters was $>0.9$ for all parameters. For the parameters modeling condition effects (magnitude effects, Fig. 10, top row), these correlations were lower for some parameters, in particular for condition effects on boundary separation and $\log (\mathrm{k})$. The likely reason is that the synthetic data were simulated from the actual posterior distribution, and there was overall little between-subject variance in some of these parameters in our data (see, e.g., Fig. 10a,f).

\section{Discussion}

We investigated the effects of a single dose of the D2-receptor antagonist haloperidol $(2 \mathrm{mg})$ on temporal discounting in a between-subjects study in a double-blind placebo-controlled setting. A diffusion model-based analysis revealed substantially smaller $\log (\mathrm{k})$ parameters and a substantial reduction in nondecision times under haloperidol versus placebo.

We applied a recent class of value-based decision models based on the DDM (Pedersen et al., 2017; Fontanesi et al., 2019; Shahar et al., 2019; Peters and D'Esposito, 2020). Comprehensive RT-based analysis was not possible in previous studies because of the specifics of task timing (Pine et al., 2010) or low trial numbers (Weber et al., 2016; Petzold et al., 2019). Model comparison confirmed previous results (Fontanesi et al., 2019; Peters and D'Esposito, 2020), such that the data were better accounted for by a model assuming a nonlinear trialwise scaling of the drift rate, and this was confirmed via posterior predictive checks of single-subject data. Extensive parameter recovery analyses confirmed that group-level parameters recovered well (Fontanesi et al., 2019; Peters and D’Esposito, 2020). Recovery of individualsubject baseline parameters (100€ magnitude condition) was excellent, whereas recovery of parameters modeling condition effects was somewhat lower. This is likely because of some parameters (e.g., boundary separation shift) showing low betweensubject variance. Modeling was further validated by the observation that drug effects were fully reproduced using a Softmax choice rule (Sutton and Barto, 1998) and by the finding that the magnitude effect (Green et al., 1997; Ballard et al., 2017; Mellis et al., 2017) was likewise replicated using the DDM-based approach. The qualitative pattern of RT effects was reproduced using a hierarchical linear regression model of trialwise inverse RTs as a function of decision conflict.

The human literature on DA and impulsivity is heterogeneous (D'Amour-Horvat and Leyton, 2014), and interpretation of these findings is complicated by several factors. First, effects of dopaminergic drugs might depend on baseline DA availability (Cools and D'Esposito, 2011), such that the same drug might impair or enhance performance in different participants, according to an inverted U-shaped function (or a different process-dependent function) (Floresco, 2013). Second, the action of D2receptor antagonists is often interpreted in terms of a reduction in DA neurotransmission (Pessiglione et al., 2006; Pine et al., 2010). But such drugs might indeed enhance DA release by predominantly binding at presynaptic DA auto-receptors, at least at lower dosages (Frank and O'Reilly, 2006) as shown in animal (Pehek, 1999; Schwarz et al., 2004) and human studies (Chen et al., 2005).

Interpretation of D2-receptor antagonist effects as a presynaptically mediated elevation of DA release might reconcile a number of conflicting results. First, our finding of reduced temporal discounting under haloperidol is in line with two recent studies that reported reduced temporal discounting following administration of D2/D3-receptor antagonists (Arrondo et al., 2015; Weber et al., 2016). On the other hand, a reduction of temporal discounting following administration of haloperidol was not observed in an earlier within-subjects study in $n=13$ participants (Pine et al., 2010) that used a slightly lower dosage of $1.5 \mathrm{mg}$ (we used $2 \mathrm{mg}$ ). Lower dosages of D2/D3-receptor antagonists might increase (rather than decrease) DA signaling (Frank and O'Reilly, 2006), an effect mediated by inhibitory feedback through presynaptic D2 auto-receptors (Grace, 1991), which may lead to an enhancement of phasic (vs. tonic) DA signaling (Frank and O'Reilly, 2006), a point that we return to below. However, we do acknowledge that such an interpretation is not general consensus in the cognitive literature on DA drug effects (Pessiglione et al., 2006; Pine et al., 2010).

Our results advance previous findings regarding the role of D2/D3-receptor antagonists in temporal discounting in several ways. First, participants performed an unrelated memory task during fMRI directly before completing the temporal discounting task. Those data revealed an overall main effect of drug condition on trial onset-related activity in caudate nucleus (Clos et al., 2019a,b) (i.e., caudate activity was increased under haloperidol). Although this neural read-out was obtained before the discounting task, both the fMRI and temporal discounting time points were well within the time of maximum haloperidol plasma levels (Franken et al., 2017). This observation is arguably more compatible with the idea that the dosage of haloperidol applied here increased (rather than decreased) striatal DA signaling. Similar neural evidence was lacking in most previous human pharmacological studies on DA effects on discounting (de Wit et 

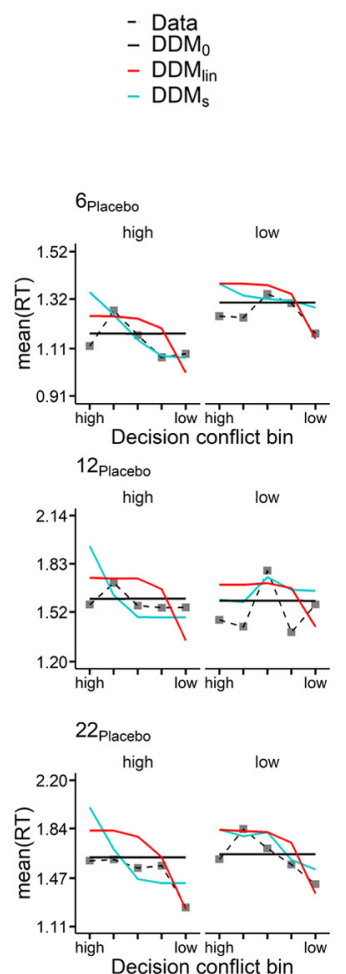

28 Placebo
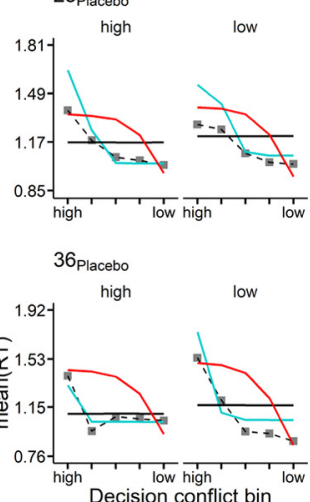

$46_{\text {Placebo }}$

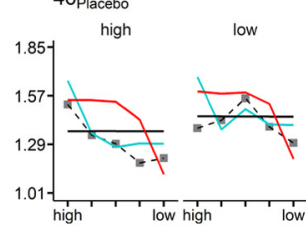

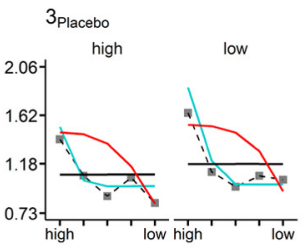
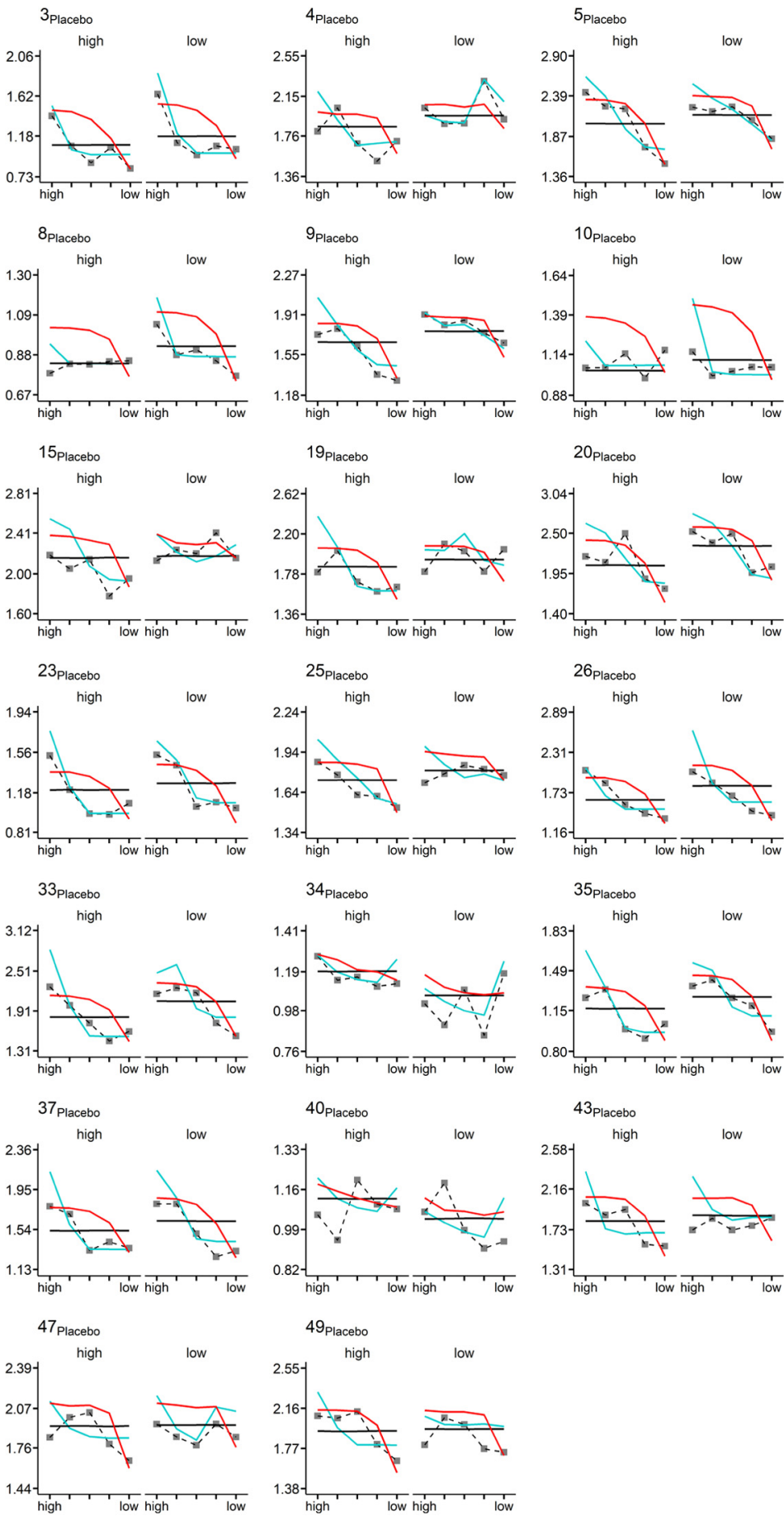

Figure 7. Placebo condition posterior predictive checks. For each participant and condition (high (left facet) represents the high magnitude condition; low (right facet) represents the low magnitude condition), trials were binned into five equal sized bins according to the absolute difference in between subjective LL and SS options (decision conflict bin). Plotted are mean observed RTs per bin (data) as well model-generated RTs (blue represents $\mathrm{DDM}_{0}$; red represents $\mathrm{DDM}_{\text {lin; }}$ orange represents $\mathrm{DDM}_{\mathrm{s}}$ ) averaged $>10,000$ datasets simulated from the posterior distribution of each hierarchical model (blue represents $\mathrm{DDM}_{0}$; red represents $\mathrm{DDM}_{\text {lin; }}$ orange represents $\mathrm{DDM}_{\mathrm{s}}$ ).

al., 2002; Hamidovic et al., 2008; Arrondo et al., 2015; Weber et al., 2016). Second, the DDM-based modeling approach adopted in the present study allowed us examine the dynamics underlying decision-making much more comprehensively than previous human pharmacological studies (de Wit et al., 2002; Hamidovic et al., 2008; Pine et al., 2010; Arrondo et al., 2015; Weber et al., 2016; Petzold et al., 2019). In addition to the drug effect on the discount rate $\log (\mathrm{k})$, diffusion modeling revealed substantially shorter nondecision times in the haloperidol group that amounted to $\approx 180 \mathrm{~ms}$ on average. Such a robust enhancement of lower-level motor and/or perceptual RT components is also more compatible with an increase, rather than a decrease, in DA transmission (Weed and Gold, 1998) and resonates with previous findings regarding a dopaminergic enhancement of RTbased response vigor (Guitart-Masip et al., 2011; Beierholm et al., 2013). An exploratory inspection of parameter correlations revealed that $\log (\mathrm{k})$ and nondecision time were positively correlated in both groups, suggesting that they might capture similar 

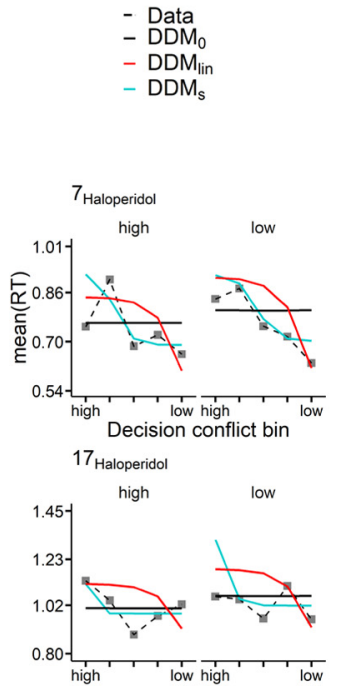

$$
24_{\text {Haloperidol }}
$$

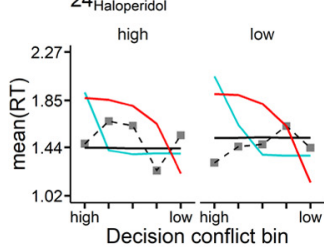

$32_{\text {Haloperidol }}$

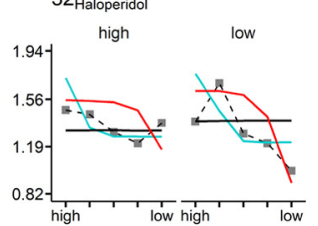

$41_{\text {Haloperidol }}$

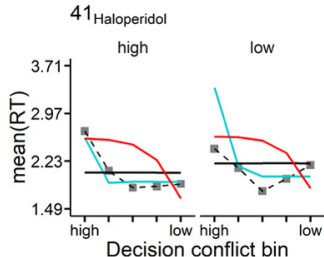

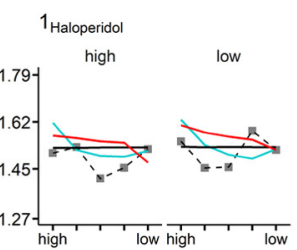
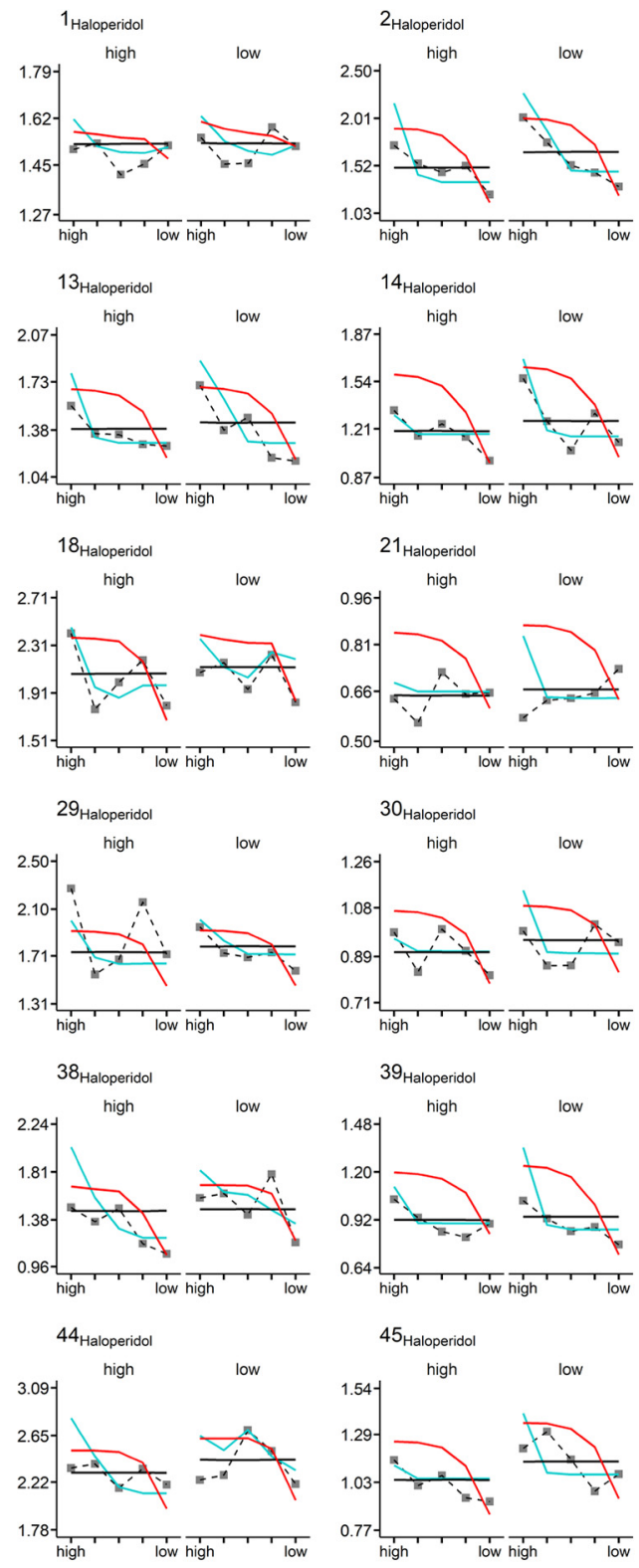
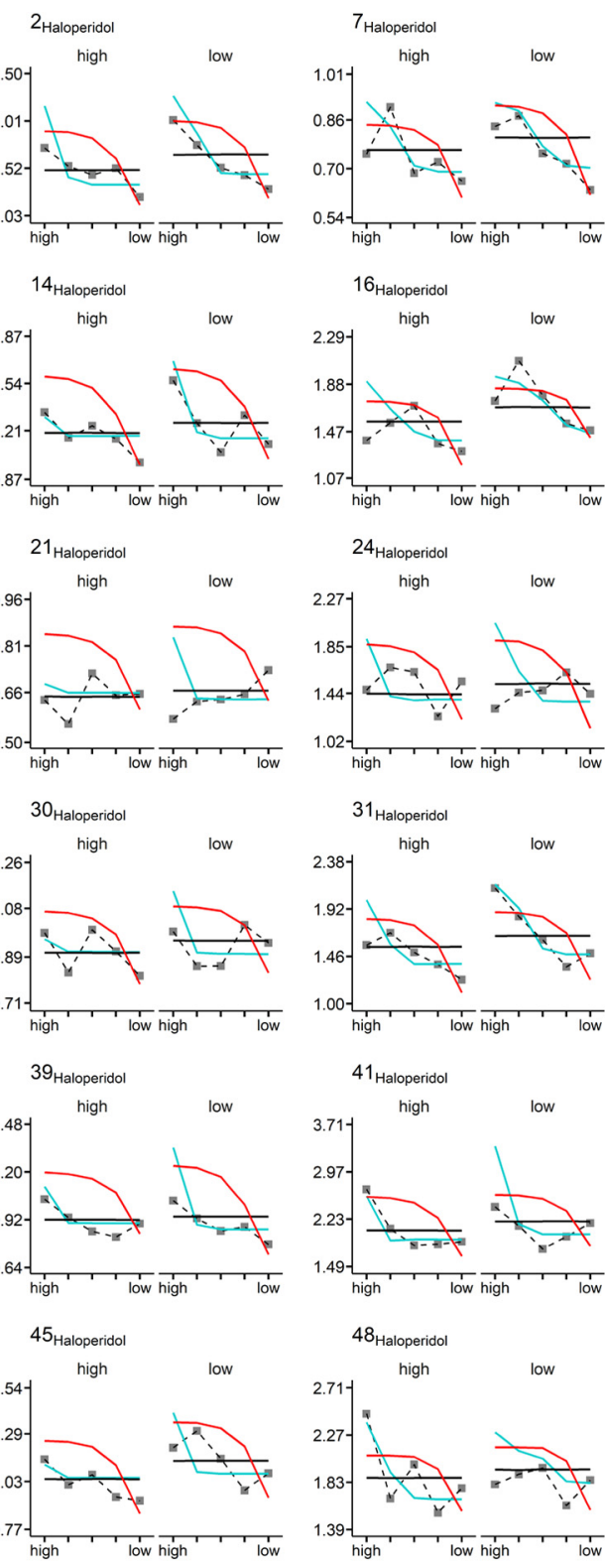

Figure 8. Haloperidol condition posterior predictive checks. For each participant and condition (high (left facet) represents the high magnitude condition; low (right facet) represents the low magnitude condition), trials were binned into five equal sized bins according to the absolute difference in between subjective LL and SS options (decision conflict bin). Plotted are mean observed RTs per bin (data) as well model-generated RTs (blue represents DDM ; red represents DDM lini $_{0}$ orange represents DDMs) averaged $>10,000$ datasets simulated from the posterior distribution of each hierarchical model (blue represents $\mathrm{DDM}_{0}$; red represents $\mathrm{DDM}_{\text {lini }}$; orange represents $\mathrm{DDM}_{s}$ ).

aspects of the data and/or might both be modulated by changes in phasic dopaminergic responses. In support of this interpretation, augmentation of DA levels in Parkinson's disease patients reduces temporal discounting (Foerde et al., 2016) and improves model-based RL (Sharp et al., 2016). Finally, this interpretation of available human D2-receptor antagonist effects would also reconcile the human and animal literature on acute dopaminergic effects on impulsivity (D'Amour-Horvat and Leyton, 2014). Together, these considerations lead us to suggest that haloperidol increased (rather than decreased) striatal DA neurotransmission, resulting in enhanced cognitive control (reduced discounting) and a substantial facilitation of motor responding (shorter nondecision times).

By what mechanism might haloperidol attenuate the impact of delay on reward valuation? According to models of basal ganglia contributions to action selection (Maia and Frank, 2011), the probability for selecting a given candidate action depends on the relative difference in activation between the direct $(g o)$ and the indirect (nogo) pathways. A similar striatal gating mechanism might underlie working memory and/or prefrontal control functions (Cools, 2011). By increasing phasic DA responses, haloperidol might increase the signal-to-noise ratio in striatal value representations, thereby increasing the likelihood that objectively smaller and/or more delayed LL rewards gain access to processing in the PFC. Naturally, other modes of action are likewise conceivable. Frontal and striatal regions are interconnected via a series of loops that follow a dorsal-to-ventral organization (Haber and Knutson, 2010), and haloperidol might impact functional interactions within these circuits (Cools, 2011), for example, related to top-down control of value representations (Hare et al., 2009, 

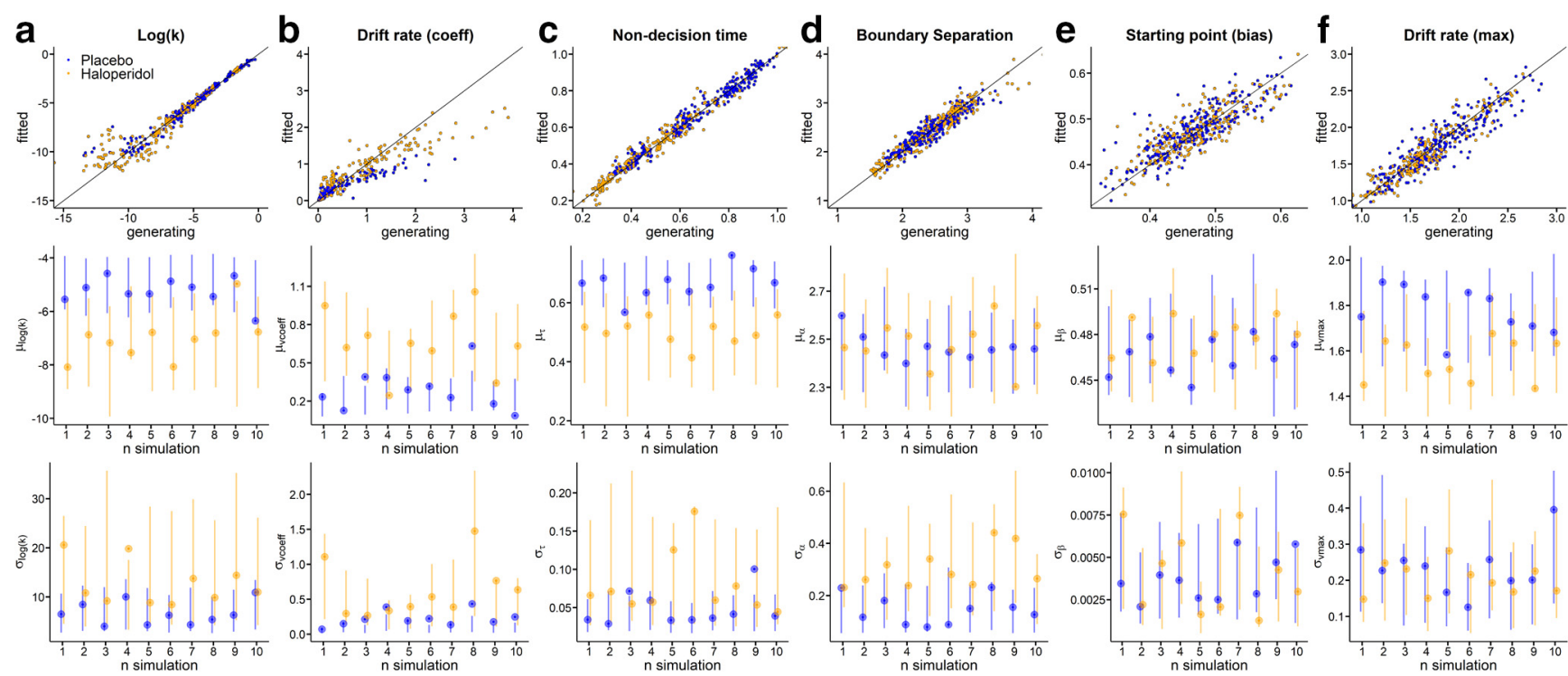

Figure 9. Parameter recovery analysis for all Baseline parameters using the $\mathrm{DDM}_{s}(\boldsymbol{a}, \log (\mathrm{k}) ; \boldsymbol{b}$, Drift rate coefficient; $\boldsymbol{c}$, Nondecision time; $\boldsymbol{d}$, Boundary separation; $\boldsymbol{e}$, Starting point bias; $\boldsymbol{f}$, Drift rate maximum). Top row: Generating parameters vs. fitted parameters for each subject across ten simulations for haloperidol group (yellow) and placebo group (blue). Second row: True generating group level hyperparameter means (points) and Bottom row: standard deviations (points) and estimated 95\% highest density intervals (lines) per fitted simulation. For correlations between generating and estimated single-subject parameters, see Extended Data Figure 9-1.
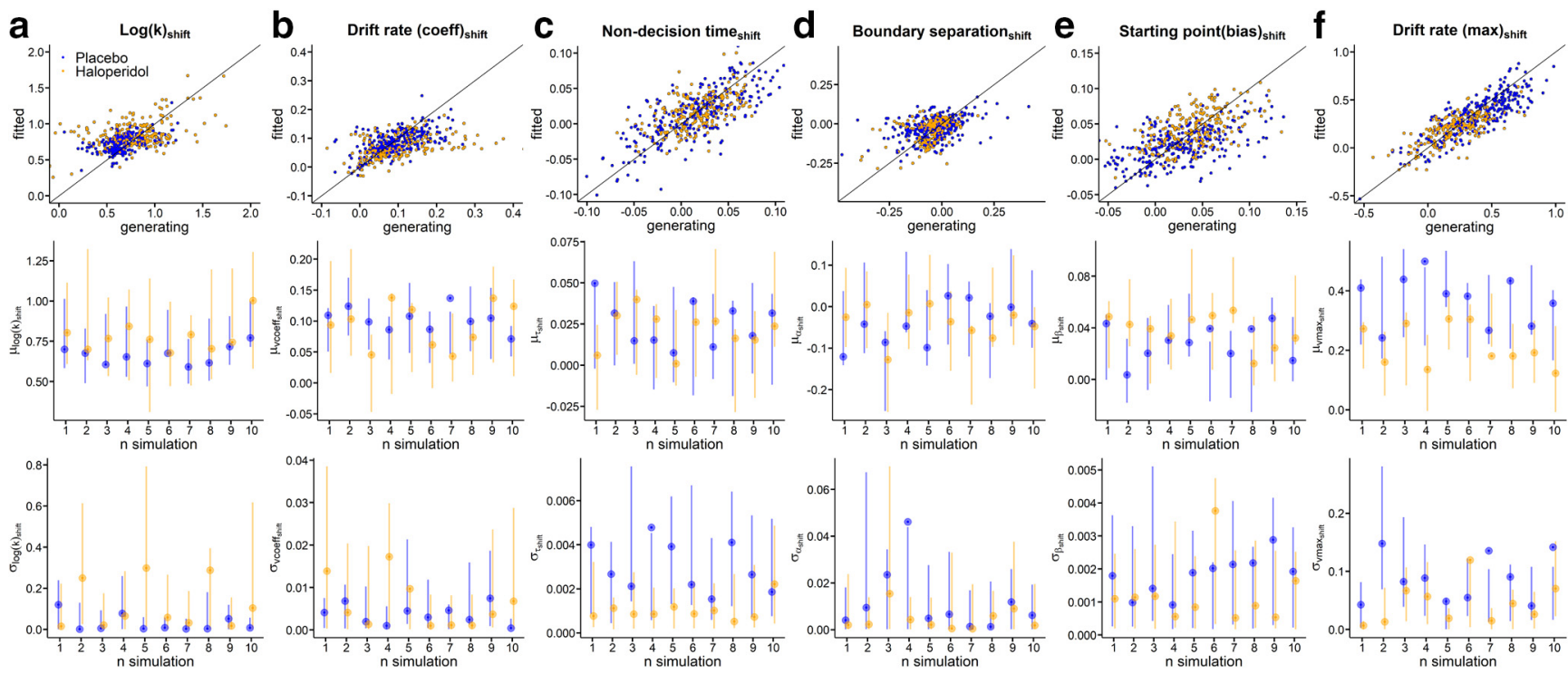

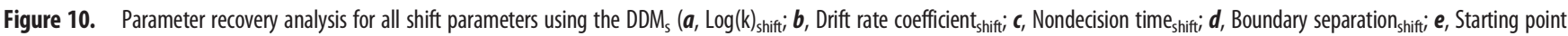

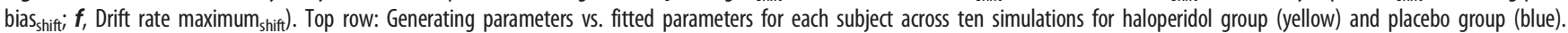
Second row: True generating group level hyperparameter means (points) and Bottom row: standard deviations (points) and estimated $95 \%$ highest density intervals (lines) per fitted simulation.

2014; Figner et al., 2010; Peters and D’Esposito, 2016). Finally, haloperidol might have directly augmented control processes in specific PFC regions (Figner et al., 2010). However, because of the much greater expression of D2 receptors in striatum compared with PFC (Seamans and Yang, 2004), it is generally assumed that prefrontal action of D2 antagonists requires substantially higher dosages than those applied in the studies examined here (Seamans and Yang, 2004; Frank and O'Reilly, 2006).

The present study has a number of limitations that need to be acknowledged. First, we did not run a within-subjects design, which would have allowed us to account for individual-participant baseline parameters in the analysis of the drug effects. Second, this also precluded us from comprehensively analyzing potential modulatory influences of, for example, individual differences in working memory on the drug effects, which might modulate DA effects on discounting (Petzold et al., 2019) and cognitive control more generally (Cools and D'Esposito, 2011). Third, the proportion of female participants was relatively large. Given the known association of ovarian hormones with the DA system (Yoest et al., 2018), future studies would benefit from testing larger sample sizes that allow for the examination of gender effects and/or from directly controlling menstrual cycle phase. Fourth, rewards were hypothetical because of the inclusion of the high-magnitude condition. However, preferences for real and hypothetical outcomes in temporal discounting tasks show a very good correspondence (Johnson and Bickel, 2002) 
and rely on similar neural circuits (Bickel et al., 2009). Also, neural haloperidol effects vary across brain regions and functions (Wächtler et al., 2020), complicating interpretation as no taskrelated imaging data were obtained here.

In conclusion, our data show that the D2-receptor antagonist haloperidol attenuated temporal discounting and substantially shortened nondecision times, as revealed by comprehensive computational modeling of choices and RTs using hierarchical Bayesian parameter estimation. These data are best accounted for by a model in which low dosages of haloperidol lead to an enhancement of phasic DA responses because of reduced feedback inhibition from D2 auto-receptors, leading to an augmentation of both lower-level (nondecision time) and higher-level (temporal discounting) decision components.

\section{References}

Acheson A, de Wit H (2008) Bupropion improves attention but does not affect impulsive behavior in healthy young adults. Exp Clin Psychopharmacol 16:113-123.

Amlung M, Petker T, Jackson J, Balodis I, MacKillop J (2016) Steep discounting of delayed monetary and food rewards in obesity: a meta-analysis. Psychol Med 46:2423-2434.

Amlung M, Marsden E, Holshausen K, Morris V, Patel H, Vedelago L, Naish KR, Reed DD, McCabe RE (2019) Delay discounting as a transdiagnostic process in psychiatric disorders: a meta-analysis. JAMA Psychiatry 76:1176.

Arrondo G, Aznárez-Sanado M, Fernández-Seara MA, Goñi J, Loayza FR, Salamon-Klobut E, Heukamp FH, Pastor MA (2015) Dopaminergic modulation of the trade-off between probability and time in economic decision-making. Eur Neuropsychopharmacol 25:817-827.

Ballard IC, Kim B, Liatsis A, Aydogan G, Cohen JD, McClure SM (2017) More is meaningful: the magnitude effect in intertemporal choice depends on self-control. Psychol Sci 28:1443-1454.

Beard E, Dienes Z, Muirhead C, West R (2016) Using Bayes factors for testing hypotheses about intervention effectiveness in addiction in addiction research. Addiction 111:2230-2247.

Beierholm U, Guitart-Masip M, Economides M, Chowdhury R, Duzel E, Dolan R, Dayan P (2013) Dopamine modulates reward-related vigor. Neuropsychopharmacology 38:1495-1503.

Bickel WK, Pitcock JA, Yi R, Angtuaco EJ (2009) Congruence of BOLD response across intertemporal choice conditions: fictive and real money gains and losses. J Neurosci 29:8839-8846.

Bickel WK, Koffarnus MN, Moody L, Wilson AG (2014) The behavioraland neuro-economic process of temporal discounting: a candidate behavioral marker of addiction. Neuropharmacology 76:518-527.

Chen YC, Choi JK, Andersen SL, Rosen BR, Jenkins BG (2005) Mapping dopamine D2/D3 receptor function using pharmacological magnetic resonance imaging. Psychopharmacology (Berl) 180:705-715.

Clos M, Bunzeck N, Sommer T (2019a) Dopamine is a double-edged sword: dopaminergic modulation enhances memory retrieval performance but impairs metacognition. Neuropsychopharmacology 44:555-563.

Clos M, Bunzeck N, Sommer T (2019b) Dopamine enhances item novelty detection via hippocampal and associative recall via left lateral prefrontal cortex mechanisms. J Neurosci 39:7920-7933.

Cools R (2011) Dopaminergic control of the striatum for high-level cognition. Curr Opin Neurobiol 21:402-407.

Cools R, D'Esposito M (2011) Inverted-U-shaped dopamine actions on human working memory and cognitive control. Biol Psychiatry 69:e113e125.

D'Amour-Horvat V, Leyton M (2014) Impulsive actions and choices in laboratory animals and humans: effects of high vs. low dopamine states produced by systemic treatments given to neurologically intact subjects. Front Behav Neurosci 8:432.

de Wit H, Enggasser JL, Richards JB (2002) Acute administration of D-amphetamine decreases impulsivity in healthy volunteers. Neuropsychopharmacology 27:813-825.

Doll BB, Simon DA, Daw ND (2012) The ubiquity of model-based reinforcement learning. Curr Opin Neurobiol 22:1075-1081.
Figner B, Knoch D, Johnson EJ, Krosch AR, Lisanby SH, Fehr E, Weber EU (2010) Lateral prefrontal cortex and self-control in intertemporal choice. Nat Neurosci 13:538-539.

Floresco SB (2013) Prefrontal dopamine and behavioral flexibility: shifting from an "inverted-U" toward a family of functions. Front Neurosci 7:62.

Foerde K, Figner B, Doll BB, Woyke IC, Braun EK, Weber EU, Shohamy D (2016) Dopamine modulation of intertemporal decision-making: evidence from Parkinson disease. J Cogn Neurosci 28:657-667.

Fontanesi L, Gluth S, Spektor MS, Rieskamp J (2019) A reinforcement learning diffusion decision model for value-based decisions. Psychon Bull Rev 26:1099-1121.

Frank MJ, O’Reilly RC (2006) A mechanistic account of striatal dopamine function in human cognition: psychopharmacological studies with cabergoline and haloperidol. Behav Neurosci 120:497-517.

Franken LG, Mathot RA, Masman AD, Baar FP, Tibboel D, van Gelder T, Koch BC, de Winter BC (2017) Population pharmacokinetics of haloperidol in terminally ill adult patients. Eur J Clin Pharmacol 73:1271-1277.

Grace AA (1991) Phasic versus tonic dopamine release and the modulation of dopamine system responsivity: a hypothesis for the etiology of schizophrenia. Neuroscience 41:1-24.

Green L, Myerson J (2004) A discounting framework for choice with delayed and probabilistic rewards. Psychol Bull 130:769-792.

Green L, Myerson J, McFadden E (1997) Rate of temporal discounting decreases with amount of reward. Mem Cognit 25:715-723.

Guitart-Masip M, Beierholm UR, Dolan R, Duzel E, Dayan P (2011) Vigor in the face of fluctuating rates of reward: an experimental examination. J Cogn Neurosci 23:3933-3938.

Haber SN, Knutson B (2010) The reward circuit: linking primate anatomy and human imaging. Neuropsychopharmacology 35:4-26.

Hamidovic A, Kang UJ, de Wit H (2008) Effects of low to moderate acute doses of pramipexole on impulsivity and cognition in healthy volunteers. J Clin Psychopharmacol 28:45-51.

Hare TA, Camerer CF, Rangel A (2009) Self-control in decision-making involves modulation of the vmPFC valuation system. Science 324:646648.

Hare TA, Hakimi S, Rangel A (2014) Activity in dlPFC and its effective connectivity to vmPFC are associated with temporal discounting. Front Neurosci 8:50.

Jackson JN, MacKillop J (2016) Attention-deficit/hyperactivity disorder and monetary delay discounting: a meta-analysis of case-control studies. Biol Psychiatry Cogn Neurosci Neuroimaging 1:316-325.

Johnson MW, Bickel WK (2002) Within-subject comparison of real and hypothetical money rewards in delay discounting. J Exp Anal Behav 77:129-146.

Kass RE, Raftery AE (1995) Bayes factors. J Am Stat Assoc 90:773-795.

Krebs CA, Reilly WJ, Anderson KG (2016) Reinforcer magnitude affects delay discounting and influences effects of $\mathrm{D}$-amphetamine in rats. Behav Processes 130:39-45.

Kroemer NB, Lee Y, Pooseh S, Eppinger B, Goschke T, Smolka MN (2019) L-DOPA reduces model-free control of behavior by attenuating the transfer of value to action. Neuroimage 186:113-125.

Lempert KM, Steinglass JE, Pinto A, Kable JW, Simpson HB (2019) Can delay discounting deliver on the promise of RDoC? Psychol Med 49:190199.

Maia TV, Frank MJ (2011) From reinforcement learning models to psychiatric and neurological disorders. Nat Neurosci 14:154-162.

Mazur JE (1987) An adjusting procedure for studying delayed reinforcement. In: Quantitative analyses of behavior (Commons ML, Mazur JE, Nevin JA, Rachlin H, eds), pp 555-573. Hillsdale, NJ: Erlbaum.

Mellis AM, Woodford AE, Stein JS, Bickel WK (2017) A second type of magnitude effect: reinforcer magnitude differentiates delay discounting between substance users and controls. J Exp Anal Behav 107:151-160.

Pedersen ML, Frank MJ, Biele G (2017) The drift diffusion model as the choice rule in reinforcement learning. Psychon Bull Rev 24:1234-1251.

Pehek EA (1999) Comparison of effects of haloperidol administration on amphetamine-stimulated dopamine release in the rat medial prefrontal cortex and dorsal striatum. J Pharmacol Exp Ther 289:14-23.

Pessiglione M, Seymour B, Flandin G, Dolan RJ, Frith CD (2006) Dopaminedependent prediction errors underpin reward-seeking behaviour in humans. Nature 442:1042-1045.

Peters J, Büchel C (2011) The neural mechanisms of inter-temporal decisionmaking: understanding variability. Trends Cogn Sci 15:227-239. 
Peters J, D’Esposito M (2016) Effects of medial orbitofrontal cortex lesions on self-control in intertemporal choice. Curr Biol 26:2625-2628.

Peters J, D'Esposito M (2020) The drift diffusion model as the choice rule in inter-temporal and risky choice: a case study in medial orbitofrontal cortex lesion patients and controls. PLoS Comput Biol 16:e1007615.

Petzold J, Kienast A, Lee Y, Pooseh S, London ED, Goschke T, Smolka MN (2019) Baseline impulsivity may moderate L-DOPA effects on valuebased decision-making. Sci Rep 9:5652.

Pine A, Shiner T, Seymour B, Dolan RJ (2010) Dopamine, time, and impulsivity in humans. J Neurosci 30:8888-8896.

Plummer M (2003) JAGS: a program for analysis of Bayesian graphical models using Gibbs sampling. In: Proceedings of the 3rd International Workshop on Distributed Statistical Computing, p 125. Technische Universit at Wien.

Robinson TE, Berridge KC (1993) The neural basis of drug craving: an incentive-sensitization theory of addiction. Brain Res Brain Res Rev 18:247291.

Schwarz A, Gozzi A, Reese T, Bertani S, Crestan V, Hagan J, Heidbreder C, Bifone A (2004) Selective dopamine D(3) receptor antagonist SB-277011A potentiates phMRI response to acute amphetamine challenge in the rat brain. Synapse 54:1-10.

Seamans JK, Yang CR (2004) The principal features and mechanisms of dopamine modulation in the prefrontal cortex. Prog Neurobiol 74:1-58.

Shahar N, Hauser TU, Moutoussis M, Moran R, Keramati M, Dolan RJ, NSPN Consortium (2019) Improving the reliability of model-based decision-making estimates in the two-stage decision task with reaction-times and drift-diffusion modeling. PLoS Comput Biol 15:e1006803.

Shakhatreh M, Jehangir A, Malik Z, Parkman HP (2019) Metoclopramide for the treatment of diabetic gastroparesis. Expert Rev Gastroenterol Hepatol 13:711-721.
Sharp ME, Foerde K, Daw ND, Shohamy D (2016) Dopamine selectively remediates "model-based" reward learning: a computational approach. Brain 139:355-364.

Shenhav A, Rand DG, Greene JD (2017) The relationship between intertemporal choice and following the path of least resistance across choices, preferences, and beliefs. Judge Decis Making 12:1-18.

Solway A, Lohrenz T, Montague PR (2017) Simulating future value in intertemporal choice. Sci Rep 7:43119.

Spiegelhalter DJ, Best NG, Carlin BP, Van Der Linde A (2002) Bayesian measures of model complexity and fit. J R Stat Soc B 64:583-639.

Sutton RS, Barto AG (1998) Reinforcement learning: an introduction. Cambridge, MA: Massachusetts Institute of Technology.

Wabersich D, Vandekerckhove J (2014) Extending JAGS: a tutorial on adding custom distributions to JAGS (with a diffusion model example). Behav Res Methods 46:15-28.

Wächtler CO, Chakroun K, Clos M, Bayer J, Hennies N, Beaulieu JM, Sommer $\mathrm{T}$ (2020) Region-specific effects of acute haloperidol in the human midbrain, striatum and cortex. Eur Neuropsychopharmacol 35:126-135.

Weber SC, Beck-Schimmer B, Kajdi ME, Müller D, Tobler PN, Quednow BB (2016) Dopamine D2/3- and $\mu$-opioid receptor antagonists reduce cueinduced responding and reward impulsivity in humans. Transl Psychiatry 6:e850.

Weed MR, Gold LH (1998) The effects of dopaminergic agents on reaction time in rhesus monkeys. Psychopharmacology (Berl) 137:33-42.

Wiehler A, Peters J (2015) Reward-based decision making in pathological gambling: the roles of risk and delay. Neurosci Res 90:3-14.

Wunderlich K, Smittenaar P, Dolan RJ (2012) Dopamine enhances modelbased over model-free choice behavior. Neuron 75:418-424.

Yoest KE, Quigley JA, Becker JB (2018) Rapid effects of ovarian hormones in dorsal striatum and nucleus accumbens. Horm Behav 104:119-129. 